\title{
Reconfigurable Binary Neural Network Accelerator with Adaptive Parallelism Scheme
}

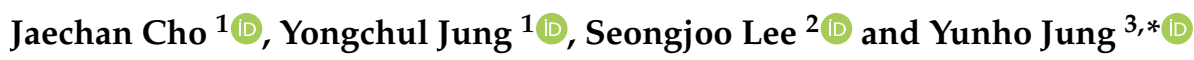 \\ 1 School of Electronics and Information Engineering, Korea Aerospace University, Goyang-si 10540, Korea; \\ jccho@kau.kr (J.C.); ycjung@kau.kr (Y.J.) \\ 2 The Department of Information and Communication Engineering and Convergence Engineering for \\ Intelligent Drone, Sejong University, Seoul 143-747, Korea; seongjoo@sejong.ac.kr \\ 3 Department of Smart Drone Convergence, School of Electronics and Information Engineering, \\ Korea Aerospace University, Goyang-si 10540, Korea \\ * Correspondence: yjung@kau.ac.kr; Tel.: +82-2-300-0133
}

Citation: Cho, J.; Jung, Y.; Lee, S.; Jung, Y. Reconfigurable Binary Neural Network Accelerator with Adaptive Parallelism Scheme. Electronics 2021, 10, 230. https://doi.org/10.3390/ electronics10030230

Academic Editor: Guido Masera

Received: 7 December 2020

Accepted: 19 January 2021

Published: 20 January 2021

Publisher's Note: MDPI stays neutral with regard to jurisdictional clai$\mathrm{ms}$ in published maps and institutional affiliations.

Copyright: (C) 2021 by the authors. Licensee MDPI, Basel, Switzerland. This article is an open access article distributed under the terms and conditions of the Creative Commons Attribution (CC BY) license (https:// creativecommons.org/licenses/by/ $4.0 /)$.

\begin{abstract}
Binary neural networks (BNNs) have attracted significant interest for the implementation of deep neural networks (DNNs) on resource-constrained edge devices, and various BNN accelerator architectures have been proposed to achieve higher efficiency. BNN accelerators can be divided into two categories: streaming and layer accelerators. Although streaming accelerators designed for a specific BNN network topology provide high throughput, they are infeasible for various sensor applications in edge AI because of their complexity and inflexibility. In contrast, layer accelerators with reasonable resources can support various network topologies, but they operate with the same parallelism for all the layers of the BNN, which degrades throughput performance at certain layers. To overcome this problem, we propose a BNN accelerator with adaptive parallelism that offers high throughput performance in all layers. The proposed accelerator analyzes target layer parameters and operates with optimal parallelism using reasonable resources. In addition, this architecture is able to fully compute all types of BNN layers thanks to its reconfigurability, and it can achieve a higher area-speed efficiency than existing accelerators. In performance evaluation using state-of-the-art BNN topologies, the designed BNN accelerator achieved an area-speed efficiency 9.69 times higher than previous FPGA implementations and 24\% higher than existing VLSI implementations for BNNs.
\end{abstract}

Keywords: artificial intelligence (AI); binary neural network (BNN); FPGA; machine learning; pattern recognition; VLSI

\section{Introduction}

Deep neural networks (DNNs) have exhibited state-of-the-art accuracy on a wide range of AI tasks, such as image classification, radar signal processing, and object detection [1,2]. Recent DNN models have become even deeper to complete tasks with higher accuracy, which requires a massive amount of computing resources and memory [3,4]. However, many resource-restricted applications call for low-cost and low-power DNN designs, and reaching a relatively lower level of accuracy is often sufficient [5]. Therefore, substantial research has been conducted to run DNNs on low-power edge devices [6-21].

An emerging trend to reduce the amount of computing resources and memory required is the use of binary neural networks (BNNs), which drop the precision of weights and activations to a single bit [15-21]. They are capable of achieving an accuracy that is on par with that of condensed full-precision DNN models, such as MobileNet [21]. BNNs can directly replace the multiply-accumulate operations by simple XNOR and popcount operations. Therefore, their greatly reduced computational workload enables the use of DNNs on edge devices, and several accelerator designs for BNN have been proposed [22-38].

BNN accelerators can be divided into two categories: streaming and layer accelerators. Streaming accelerators are designed for all or most layers in a target network [22-33]. Since 
optimized hardware is implemented for each layer, this type of architecture usually offers reasonable latency. However, streaming accelerators require more resources than layer accelerators, which are implemented for a specific target layer. In addition, they can only support the network topology that is targeted prior to implementation. In other words, if a network is optimized and implemented for a specific application, it needs to be structurally changed and re-designed to use it in other applications. Therefore, BNNs with a streaming architecture cannot always satisfy resource constraints and are thus infeasible for various sensor applications in edge AI because of their complexity and inflexibility [20].

On the other hand, layer accelerators are designed to handle a target layer in BNN topologies [34-38]. Therefore, these architectures require less resources than streaming accelerators, which are designed for all or most of the layers of a target network topology. In addition, since layer accelerators need to be able to cope with various types of layers, that is layers with different input feature map (fmap) sizes and different numbers of in/out channels, most layer accelerators have been designed with reconfigurable architectures that can handle various network topologies. Therefore, this type of architecture can support various sensor applications and is well suited for resource-constrained applications.

Conventional layer accelerator designs usually fall under one of two categories according to how data scheduling is implemented: filter-level parallelism and fmap-level parallelism. Accelerators with filter-level parallelism are designed with a line-buffer architecture that stores the previous row of the input fmap for sliding operations [34-36]. Such architectures offer efficient data reuse and higher convolution throughput. They have been used widely in convolution-based image processing applications. However, typical network topologies consist of layers having varied structures ranging from an initial convolutional (CONV) layer to deep fully connected (FC) layers, and each layer can have a different architecture. In general, the number of in/out channels gradually increases in the deeper layers, whereas the fmap size becomes smaller. Therefore, accelerators with filter-level parallelism, which is optimized for CONV operations, cannot attain higher utilization and throughput in deep layers that are similar to FC layers. In addition, these accelerators require more resources than other types of architectures to store previous row data and integer-scale intermediate values of the popcount operation.

On the other hand, several accelerators that introduce fmap-level parallelism have been proposed $[37,38]$. Accelerators with fmap-level parallelism load and process data on a per-channel basis, with $100 \%$ utilization and throughput at deeper layers exceeding a certain number of input channels. In addition, these architectures require less resources than those with filter-level parallelism because they can rapidly binarize intermediate results via efficient data scheduling [37]. However, the throughput of these accelerators is excessively degraded in the initial layers having a small number of channels.

In this paper, we propose a reconfigurable $\mathrm{BNN}$ accelerator with adaptive parallelism that offers high throughput performance in all layers. The proposed accelerator analyzes target layer parameters and adaptively applies parallelism schemes, which consist of fmap-level parallelism combined with an advanced method. This architecture can perform BNN operations with a reasonable amount of resources because the supported parallelism schemes are based on the mechanisms of fmap-level parallelism. The design and implementation results of the proposed architecture are also presented. The remainder of this paper is organized as follows. Section 2 briefly reviews BNNs and related works. Section 3 describes the proposed adaptive parallelism scheme and presents performance evaluation results. Section 4 discusses the hardware architecture and implementation results. Finally, Section 5 concludes the paper. 


\section{Background}

\subsection{Binary Neural Network}

The basic computation at each layer of a convolutional neural network (CNN) can be expressed as:

$$
\mathbf{y}\left(c_{\text {out }}\right)=\mathbf{f}\left(\sum_{c_{\text {in }}}\left(\mathbf{W}\left(c_{\text {out }}, c_{\text {in }}\right) \otimes \mathbf{x}\left(c_{\text {in }}\right)\right)+\mathbf{b}_{c_{\text {out }}}\right),
$$

where $\mathbf{y}, \mathbf{x}, \mathbf{W}$, and $\mathbf{b}$ are the output, input fmaps, weights, and biases, respectively. $c_{i n}$ and $c_{\text {out }}$ are the number of in/out fmaps (in/out channels) in high-dimensional convolutions, as shown in Figure 1. The output fmaps, which have a size of $c_{\text {out }} \times h_{\text {out }} \times w_{\text {out }}$, can be obtained through a convolution operation between the input fmaps and the filters, which is the set of weights. $\mathbf{f}$ is a nonlinear activation function, which is typically applied after each CONV layer or FC layer. These functions are usually conventional nonlinear functions, such as the rectified linear unit (ReLU), sigmoid, and hyperbolic tangent [6].
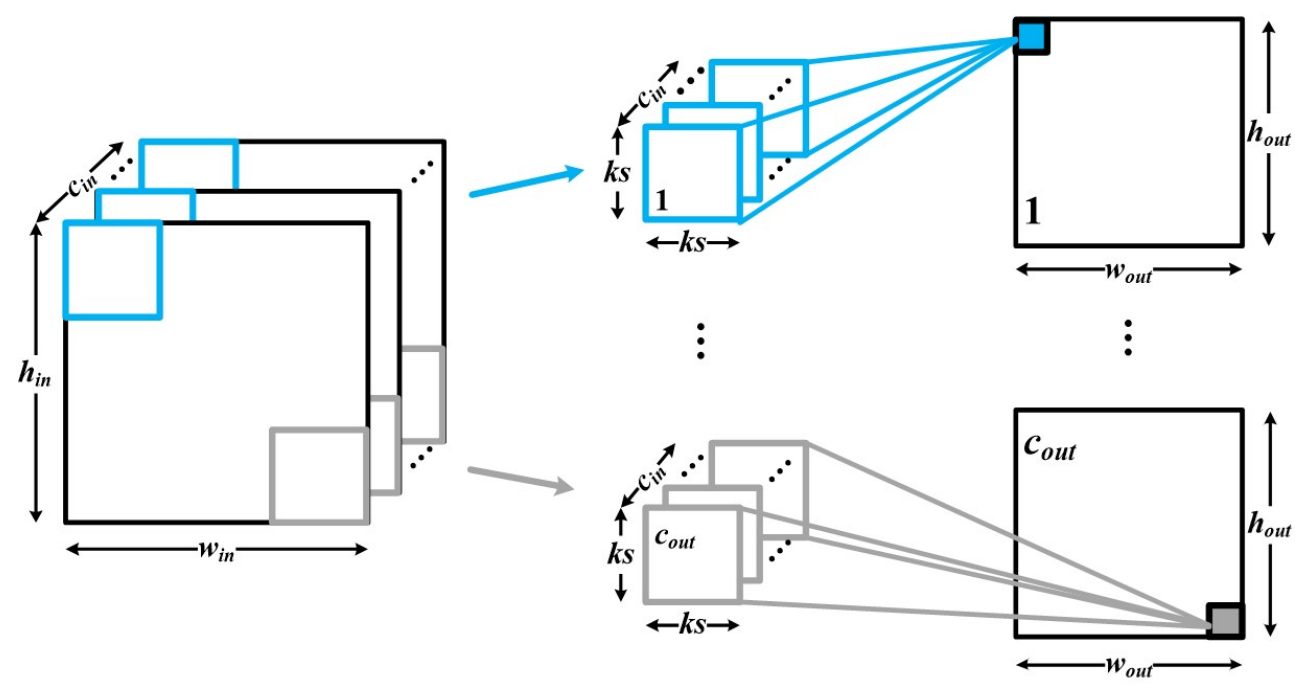

Figure 1. High-dimensional convolutions in CNNs.

In addition, various optional layers can be found in the typical network topologies of $\mathrm{CNNs}$, such as the pooling and normalization layers. Pooling reduces the dimensionality of the fmaps and is applied to each fmap separately. In batch normalization (BN), the output of the activation function is further scaled and shifted as follows:

$$
\mathrm{BN}(k)=\gamma \frac{k-\mu}{\sigma}+\beta,
$$

where $\gamma, \beta, \mu$, and $\sigma$ are learned parameters and $k$ is the input of the BN function.

In BNNs, $\mathbf{f}$ in (1) can be expressed as $\operatorname{sign}(\mathrm{BN}(k))$, which is the binarization of the $\mathrm{BN}$ output [20]. Since the values of $\mathbf{y}, \mathbf{x}, \mathbf{W}$, and $\mathbf{b}$ are +1 or $-1, \otimes$ can be reduced to bitwise operations. If the binary values obtained from these operations are encoded with +1 as a one-valued bit and -1 as a zero-valued bit, a multiplication operation is equivalent to an XNOR logical operation on the binary values. The sum of XNOR operations can also be calculated via a simple popcounting, that is counting the number of bits set to one. In addition, the costly calculation of $\operatorname{sign}(\mathrm{BN}(k)))$ can be reorganized into:

$$
\operatorname{sign}(\mathrm{BN}(k))=\left\{\begin{array}{ll}
1 & \text { if } k \geq \sigma \beta / \gamma-\mu, \text { else } 0(\text { when } \sigma / \gamma<0) \\
1 & \text { if } k \leq \sigma \beta / \gamma-\mu, \text { else } 0(\text { when } \sigma / \gamma>0)
\end{array} .\right.
$$

Multiplication and division in (2) can be replaced with a simple comparison with a threshold $\tau \doteq \sigma \beta / \gamma-\mu$, defined for convenience. 
In the training phase, the pooling layers in the BNNs are generally placed before the activation layer because of their higher accuracy. However, in the inference of BNNs, there is no loss of accuracy caused by the different ordering of these layers [20]. Since the max pooling of encoded bits as one or zero can be achieved using simple OR logical operations, placing the pooling layer after the activation layer is more efficient in the inference phase.

\subsection{BNN Acceleration Hardware}

BNNs have a greatly reduced computational workload and enable the use of DNNs on edge devices. To reach higher efficiency, several accelerator designs for BNNs have been proposed [22-38]. They can be divided into two categories: streaming and layer accelerators. Streaming accelerators are designed for all or most layers in a target network [22-33]. One of the earliest streaming architectures is FINN [22], which outperforms the conventional FPGA based deep inference accelerators. FINN is also extended to FINN-R [23] and BNNspli [24] to reach the higher performance of BNN inference. In [25,26], the FPGA based streaming accelerators were designed and compared with an ASIC , CPU, and GPU.

The streaming architectures with advanced design methods were also proposed [27-30]. SimBNN [27] analyses the data similarities to significantly reduce the operational complexity, and ReBNet [28] uses residual binarizations for efficient hardware implementation in ResNet architectures. LP-BNN [29] adopts layer parallelism and supports nearly perfect load balancing for various types of BNNs. In [30], a fully digital ASIC architecture with computation tightly coupled to the memory for aggressive data reuse was proposed. In addition, several streaming accelerators [31-33] apply improved learning schemes for BNNs. Streaming architectures can offer high throughput performance because optimized hardware is implemented for each layer. However, this type of architecture cannot always satisfy resource constraints and is thus infeasible for various sensor applications in edge AI because of the complexity and inflexibility [20].

On the other hand, layer accelerators are designed to handle a target layer in BNN topologies [34-38]. Conventional layer accelerator designs usually fall under one of two categories according to how data scheduling is implemented: filter-level parallelism and fmaplevel parallelism. Accelerators with filter-level parallelism are designed with a line-buffer architecture that stores the previous row of the input fmap for sliding operations [34-36]. In [34], a batch normalization-free technique was proposed, which can use a simple comparison instead of complex operations. FBNA[35] focuses on binarizing the first layer in BNNs and reduces the processing time for the first layer. In [36], a BNN on FPGA was proposed, which uses on-chip memories only. Although accelerators with filter-level parallelism offer efficient data reuse and higher throughput for CONV layers, they cannot attain higher utilization and throughput in deep layers such as FC layers. In addition, these accelerators require more resources than other types of architectures to store the previous row of the input fmap and integer-scale intermediate values of the popcount operation.

In contrast, several accelerators that introduce fmap-level parallelism have been proposed [37,38]. Accelerators with fmap-level parallelism load and process data on a per-channel basis, with $100 \%$ utilization and throughput at deeper layers exceeding a certain number of input channels. In [37], the XNOR neural engine (XNE) was proposed, which adopts fmap-level parallelism and a tightly coupled shared memory paradigm. In [38], a design automation scheme for BNNs with fmap-level parallelism was proposed for near-sensor processing. The accelerators with fmap-level parallelism require less resources than those with filter-level parallelism because they can rapidly binarize intermediate results via efficient data scheduling [37]. However, the throughput of these accelerators is excessively degraded in the initial layers having a small number of channels.

\section{Proposed Parallelism Scheme}

3.1. Proposed Parallelism Scheme of the BNN Accelerator

The proposed BNN accelerator adaptively applies two parallelism schemes according to the architectures of the target layer to offer high throughput in all layers. Our adaptive 
parallelism scheme consists of a combination of fmap-level parallelism and an advanced method that can efficiently cope with certain CONV layers that fmap-level parallelism cannot handle with $100 \%$ utilization. To minimize resource requirements, the proposed BNN accelerator is designed to operate with two parallelism schemes while using a similar data path as in conventional architectures with fmap-level parallelism. In addition, the proposed BNN accelerator is designed to make efficient use of the memory bandwidth allowed in various SoC platforms by using the design parameter $P_{h w}$, which is the maximum number of operations that can be processed in one clock cycle. In other words, if $P_{h w}$ is set according to the memory bandwidth allowed in each SoC, our architecture can load $P_{h w}$-size data in one clock cycle.

The first parallelism scheme (i.e., the advanced method) shown in Figure 2 is implemented for the initial layers, which have a small number of channels. The data scheduling of this scheme loads and processes $k s \times P_{\text {cin }}$ data bits per cycle, where $k s$ is the kernel size and $P_{c i n}$ is the number of fmaps that can be loaded and processed in one clock cycle. Figure 3 shows how CONV layers are reorganized in the first parallelism mode, where $i_{c}$ and $o_{c}$ mean the number of input and output channels, respectively. The loops are reordered differently than in conventional BNN loops, bringing a kernel width loop and an input fmap loop to the innermost position. These inner loops are computed in the proposed BNN accelerator in one clock cycle. The in/out fmap loops split into an inner loop (computing units on processing elements; $P_{c i n}$ for input and $P_{h w}$ for output fmaps) and an outer loop (cycling iterations on network control unit; $c_{i n} / P_{c i n}$ for input and $c_{o u t} / P_{h w}$ for output fmaps). This scheme can efficiently cope with certain CONV layers in which utilization is drastically degraded when using fmap-level parallelism, as detailed in Section 3.2.2.
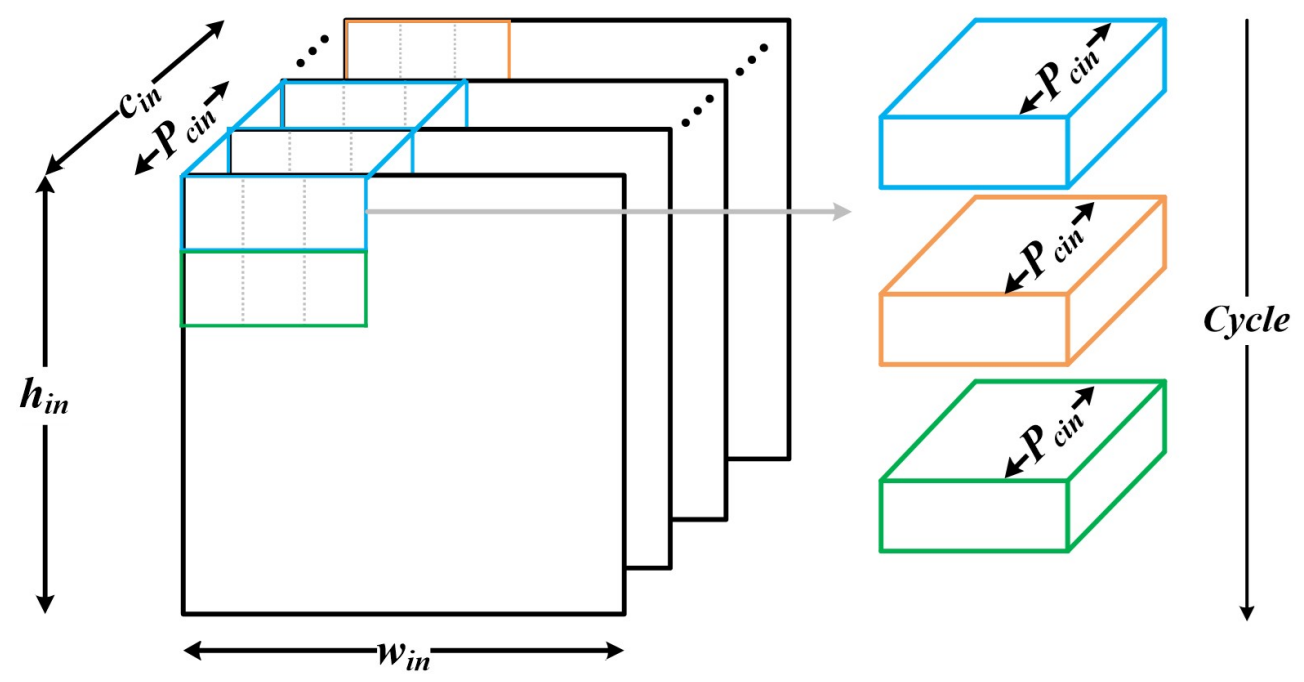

Figure 2. Data scheduling example of the first parallelism scheme.

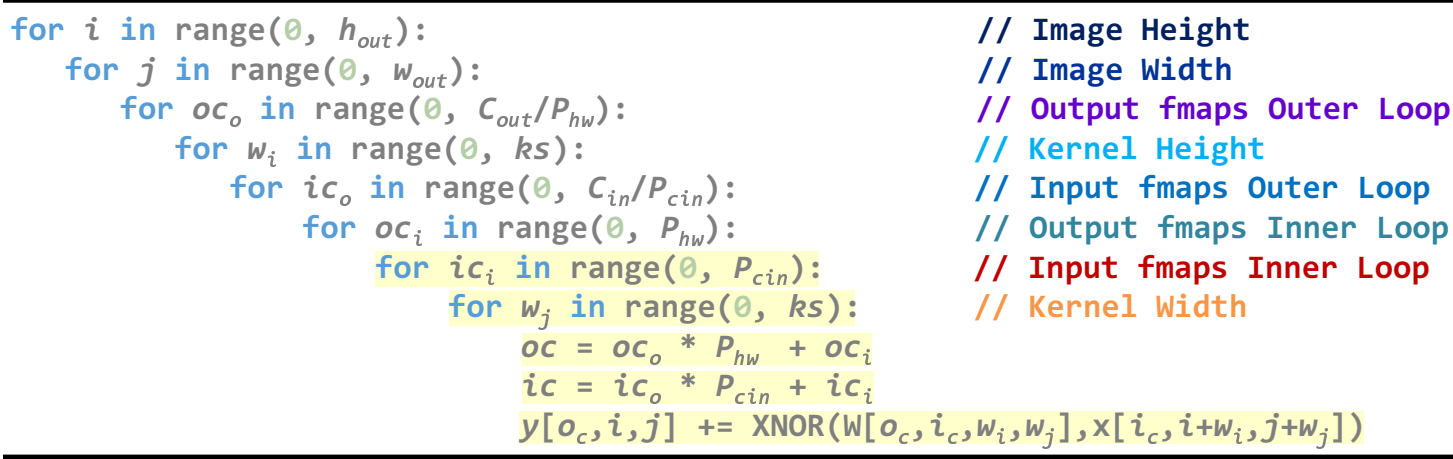

Figure 3. BNN layers' loop for the first parallelism scheme; the loops highlighted in light yellow are computed in one clock cycle. 
The second parallelism scheme used in the proposed BNNs accelerator is described in Figures 4 and 5. Similar to fmap-level parallelism, this scheme loads and processes data on a per-channel basis, and it can achieve $100 \%$ utilization at deeper layers exceeding a certain number of input channels. An overview of this parallelism scheme is shown in Figure 5. The kernel width loop is moved outward, and the hardwired inner loop in the accelerator is only focused on the fmap channels. The parallelism parameter $P_{h w}$ is used to define the number of simultaneous XNOR operations in the processing elements (PEs) per cycle. In other words, $P_{h w}$ input fmap channels can be loaded and processed in one clock cycle. Therefore, the data scheduling of this scheme handles more channels per cycle than other schemes in deeper layers.
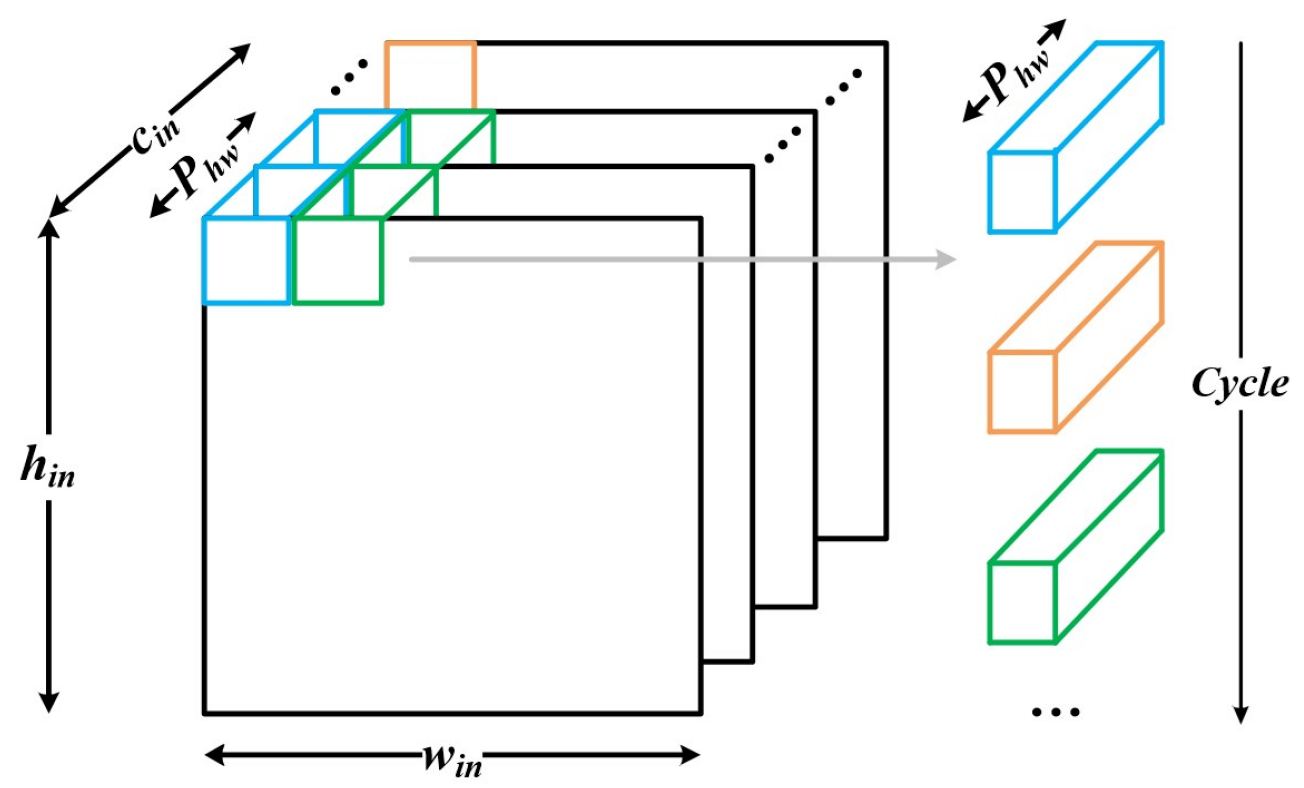

Figure 4. Data scheduling example of the second parallelism scheme.

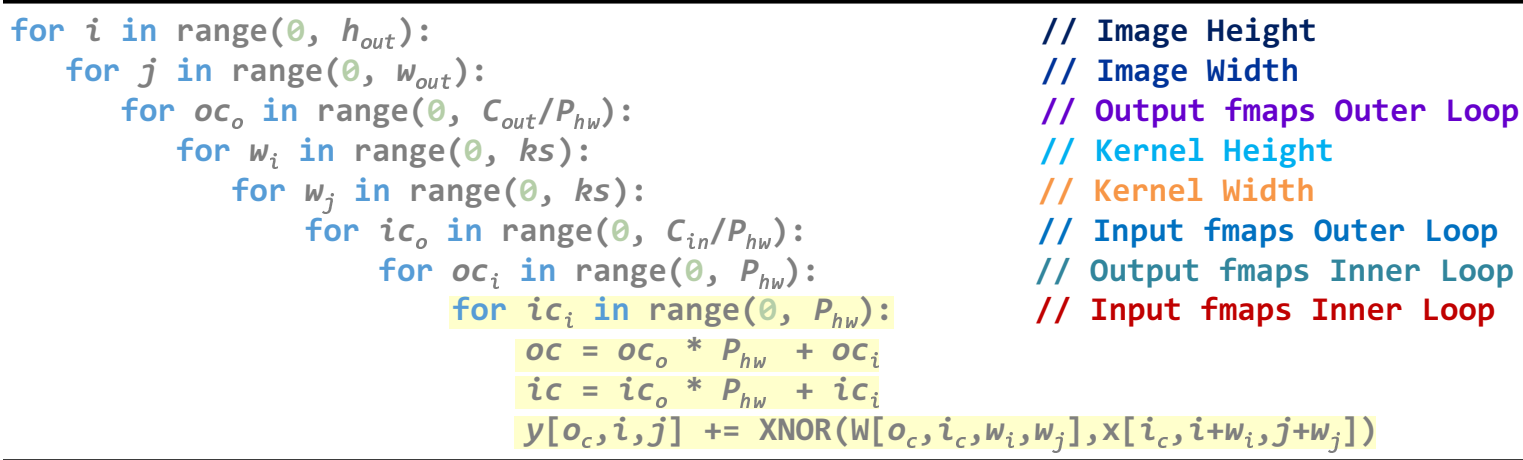

Figure 5. BNN layers' loop for the second parallelism scheme; the loops highlighted in light yellow are computed in one clock cycle.

The proposed BNN accelerator executes one of the two parallelism schemes depending on the target layer as follows:

$$
m_{t}=\left\{\begin{array}{ll}
0, & c_{i n}<P_{h w} \\
1, & c_{i n} \geq P_{h w}
\end{array},\right.
$$

where $m_{t}$ is the parallelism mode at the current target layer. The accelerator performs each parallelism scheme according to the value $m_{t}$, as shown in Table 1. Considering that the 
processor is designed with $P_{h w}=256$, if it targets a layer with respective $c_{i n}$ and $k s$ values of 128 and three, the first parallelism is used, and the accelerator attains $75 \%$ utilization. This is a utilization improvement of $25 \%$ compared with the $50 \%$ attained via fmap-level parallelism. In contrast, if $c_{\text {in }}$ is 256 or more, the proposed accelerator operates based on the second parallelism scheme, which can achieve $100 \%$ utilization.

Table 1. Supported parallelism schemes based on $m_{t}$ of the proposed BNN accelerator.

\begin{tabular}{cc}
\hline Mode & Parallelism Scheme \\
\hline$m_{t}=0$ & First scheme introduced in Figure 2 \\
$m_{t}=1$ & Second scheme shown in Figure 4 \\
\hline
\end{tabular}

\subsection{Simulation Results}

\subsubsection{Target Network Topologies}

To evaluate the efficiency of the proposed accelerator, common topologies of BNNs were used to make performance comparisons [20], as listed in Table 2. The architectures are described layer-by-layer using our own notation. Here, 2C128 and 2FC1024 refer to two CONV layers and two FC layers, with 128 and 1024 output channels, respectively. All max pooling (MP) layers have a size of $2 \times 2$ and a stride of two. The first topology is the original BNN described by Courbariaux et al. [16] and is referred to as BNN-Cifar10 in this paper. It is a variation of the VGG-11 topology with six CONV layers and three FC layers, as shown in Figure 6, and was used on the CIFAR-10 datasets. The CIFAR-10 dataset consists of 60,000 $32 \times 32$ photos and contains 10 different classes, six different animals and four different vehicles. BNN-Cifar10 delivers state-of-the-art accuracy on the CIFAR-10 dataset. The second topology used for evaluation is the well-known VGG-16 model for the ImageNet dataset. The ImageNet dataset consists of 1.2 million images and contains 1000 different classes.

Table 2. Architectures of the network topologies used to evaluate performance. MP, max pooling; $\mathrm{C}$, CONV.

\begin{tabular}{cccc}
\hline Network & Architecture & Dataset & Accuracy \\
\hline BNN-Cifar10 & 2C128-MP-2C256-MP-2C512-MP-2FC1024-FC10 & CIFAR-10 & $89.95 \%$ \\
\hline VGG-16 & $\begin{array}{c}\text { 2C64-MP-2C128-MP-3C256-MP-3C512-MP- } \\
\text { 3C512-MP-2FC4096-FC1000 }\end{array}$ & ImageNet & $75.5 \%$ \\
\hline
\end{tabular}

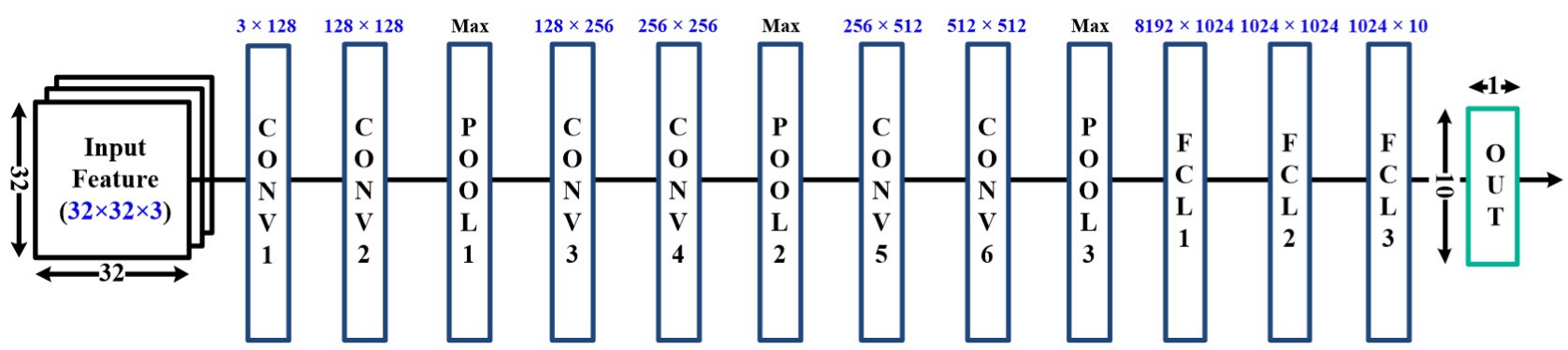

Figure 6. Topology of the BNN designed for CIFAR-10.

\subsubsection{Performance Evaluation Results}

We used operations per cycle (ops/cycle) to compare the throughput performance of our accelerator with that of existing BNN architectures. This parameter refers to the number of operations the accelerator can process per clock cycle, and we counted XNOR and popcount as separate operations [37]. In addition, ops/cycle can also represent the memory access efficiency because the number of operations that can be processed in each 
cycle is related to the number of data that can be loaded from memory in each cycle. To evaluate performance, we compared efficient BNN layer accelerators targeted for a lowpower microcontroller [34-38]. To make a fair comparison, a similar number of operators (XNOR gates and adders for popcounts) were assumed for each accelerator. In addition, the first layer, whose fmaps are normally floating-point image data, was excluded in the comparison.

First, our architecture was assumed to have $P_{h w}=256$, and Reference [34] assumed having a similar number of operators (252). Moreover, Reference [34] adopted filter-level parallelism, which significantly degrades throughput performance in deeper layers, as shown in Figure 7. Although the work in [34] showed excellent peak performance (Gops/s, giga operations per second), it used more resources than the proposed accelerators, as detailed in Section 4.2.1, and the throughput decreased in other layers because it applied an inflexible parallelism scheme. The proposed parallelism can provide higher throughput performance in almost all layers (C4 FC3), as shown in Figure 7 . In addition, when comparing at $150 \mathrm{MHz}$, which was the operating frequency of the work in [34], the proposed accelerator operates with a lower latency of $1.57 \mathrm{~ms}$ for BNN-Cifar10 and of $30.92 \mathrm{~ms}$ for VGG-16 compared with [34].

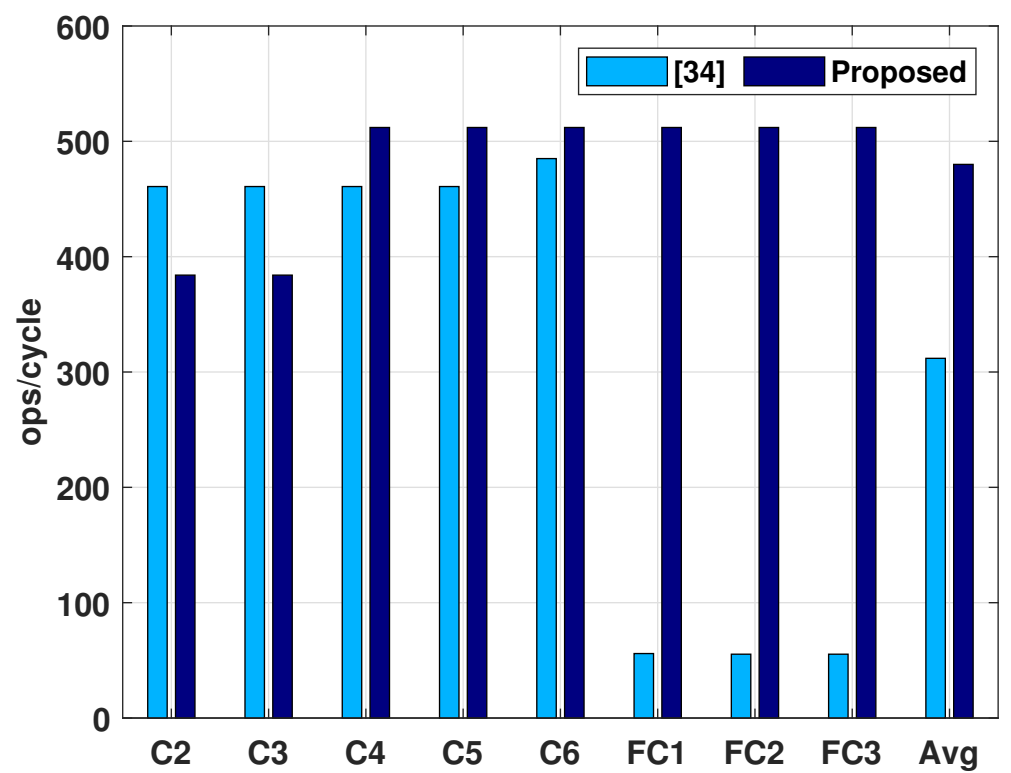

Figure 7. Comparison results of throughput performance for BNN-Cifar10. ops, operations.

On the other hand, the XNOR neural engine (XNE) [37] uses fmap-level parallelism to achieve $100 \%$ efficiency in the deeper layers, but its performance decreases sharply in the upper CONV layers, as shown in Figure 8. Our architecture and XNE were assumed to have $P_{h w}=256$ to make a fair comparison. The proposed accelerator can achieve on average ops/cycle 51.2 higher than XNE for VGG-16 and 32 higher for BNN-Cifar10. In addition, it can process with a lower latency of $1.96 \mathrm{~ms}$ for BNN-Cifar10 and $120.42 \mathrm{~ms}$ for VGG-16 compared with XNE when operating at a clock frequency of $300 \mathrm{MHz}$, which is the operating frequency of [37]. 


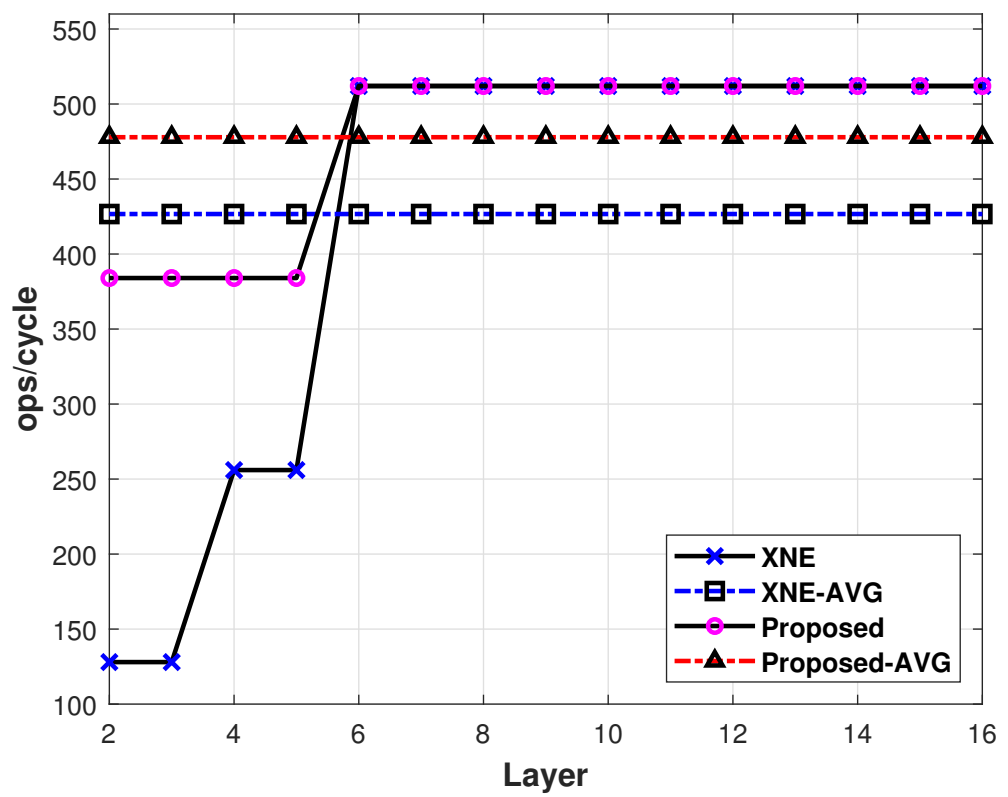

Figure 8. Comparison results of throughput performance for VGG-16. XNE, XNOR neural engine.

\section{Design and Implementation Results}

\subsection{Hardware Architecture}

Figure 9 shows the block diagram of the proposed BNN accelerator, including a network control unit (NCU), processing elements (PEs), a popcount unit (PCU), an accumulator, an activation unit, and a pooling unit. The PEs are composed of XNOR gates, AND gates, and a sub-popcount unit for processing 8 bit words. The feature vector that is input from the system is stored in a feature register for reuse. This feature vector is multiplied by the weight stream by the $P_{h w}$ XNOR gates in the PEs. To allow the proposed processor to operate with a smaller number of input features than $P_{h w}$, the product vector is masked by sum filter coefficients, in an array of AND gates.

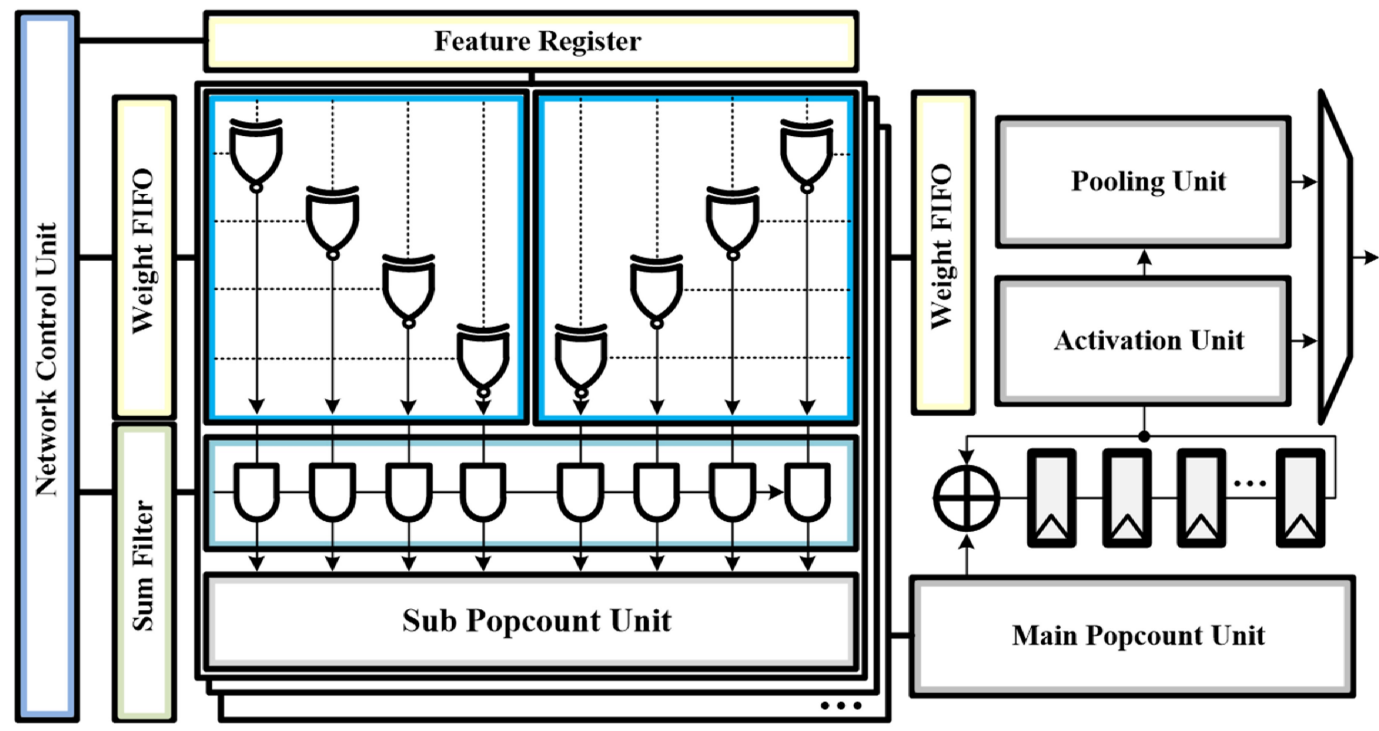

Figure 9. Block diagram of the proposed BNN accelerator.

A popcount unit based on a simple combinational circuit is used to count the number of " $1 \mathrm{~s}$ " in the unmasked vector. Each PE has a sub-popcount unit for 8 bit words, from which a 4 bit output that ranges from zero to eight is extracted. These output components are merged in the main popcount unit. The final count is accumulated with the output of the current register in the accumulator. The accumulator consists of adder and $P_{h w}$ 
registers, and each register stores one output computed in a full accumulation cycle. The accumulated values are binarized in the activation unit with the thresholding phase defined in (3). This simple comparison involves batch normalization and binarization, as mentioned in Section 2.1. The max pooling of binarized values is computed using OR logical operations in the pooling unit when a target layer has a pooling phase. Otherwise, the binarized values bypass the pooling unit for flexibility.

The NCU is designed to execute the proposed parallelism schemes. It has R/Wregisters for loading the parameters of the target layer (e.g., $c_{\text {out }}, h_{\text {out }}, w_{\text {out }}, k s$, etc.), a simple finitestate machine (FSM) to iterate the loop execution, and address calculators. The main FSM orchestrates the operation of the proposed accelerator and communicates with the microcontroller unit (MCU). By analyzing the layer parameters and selecting an appropriate parallelism scheme, the NCU controls the data loading phase and generates a memory address. It loads the data until the operation required for the current operation is completed, and the intermediate results during each iteration are stored in the accumulator. After these phases are completed, the threshold values from (3) are loaded and compared with the accumulated values in the activation unit. Then, the final outputs are streamed out to the bus interface.

\subsection{Implementation Results}

\subsubsection{FPGA Implementation Results}

The proposed BNN accelerator was designed using the Verilog hardware Description Language (HDL) and implemented on an XCZU7EV FPGA targeting the Xilinx ZYNQ Ultrascale+ ZCU-104 evaluation board. The proposed architecture with $P_{h w}=256$ requires 1050 configurable logic blocks (CLBs), 4816 CLB LUTs, and 2 DSPs, as shown in Table 3. In addition, the accelerator with the proposed parallelism schemes can process at an operating frequency of $371.6 \mathrm{MHz}$. To evaluate the efficiency of our architecture, we compared it with previous BNN implementations targeted at FPGA devices [34,35]. As shown in Table 3, although the proposed accelerator exhibits a lower peak performance than previous designs, it offers higher efficiency in terms of area (Gops/s/KLUT, Gops/s/KCLB) and power $(\mathrm{Gops} / \mathrm{s} / \mathrm{W})$. The accelerators with filter-level parallelism $[34,35]$ require more resources than the proposed accelerator because they need to store the previous row data and integer-scale intermediate values of the popcount operation.

Table 3. Comparison of the FPGA implementation results of our accelerator with previous works. CLB, configurable logic block.

\begin{tabular}{cccc}
\hline & [34] & [35] & Proposed $\left(P_{h w}=\mathbf{2 5 6}\right)$ \\
\hline FPGA & XCZU9EG & XC7Z020 & XCZU7EV \\
\hline Target Network & VGG-16 & BNN-Cifar10 & VGG-16 \\
\hline Parallelism & \multicolumn{2}{c}{ Filter-level } & Proposed \\
\hline Operating Frequency & $150 \mathrm{MHz}$ & $143 \mathrm{MHz}$ & $371 \mathrm{MHz}$ \\
\hline CLB (Slice) & 47,946 & N.A. & 1050 \\
\hline LUT & N.A. & $34.9 \mathrm{~K}$ & $4.8 \mathrm{~K}$ \\
\hline DSP & 4 & 0 & 2 \\
\hline BRAM & 1367 & 103 & 89 \\
\hline Peak Performance & $460.8 \mathrm{Gops} / \mathrm{s}$ & $722.8 \mathrm{Gops} / \mathrm{s}$ & $177.68 \mathrm{Gops} / \mathrm{s}$ \\
\hline \multirow{2}{*}{ Gops/s/KLUT } & N.A. & 21 & 37.02 \\
\cline { 2 - 4 } Gops/s/KCLB & 9.61 & N.A. & 169.22 \\
\hline Power Efficiency (Gops/s/W) & 20.94 & 219 & 250
\end{tabular}




\subsubsection{VLSI Implementation Results}

The proposed BNN accelerator was also synthesized to gate-level circuits using a $40 \mathrm{~nm}$ CMOS standard cell library. The key features of the proposed BNN accelerator are summarized in Table 4 . It can be seen that the proposed architecture requires $23.37 \mathrm{~K}$ logic gates with a total die size of $0.016 \mathrm{~mm}^{2}$. This system can operate at an operating frequency of $450 \mathrm{MHz}$, and real-time processing is possible. To evaluate the efficiency of our architecture, we compared it with XNE [37] and XNORBIN [30], which represent the VLSI implementation results for the BNNs. To make a fair comparison, our architecture and XNE were assumed to have $P_{h w}=128$, and we normalized the area as:

$$
A_{\text {norm }}=\frac{\text { Area }}{(\text { Tech } / 40 \mathrm{~nm})^{2}},
$$

where Tech is the process technology expressed in nanometers [39]. As shown in Table 4, the proposed accelerator exhibited an average area-speed efficiency (Gops/s $/ A_{\text {norm }}$ ) $24 \%$ higher than XNE with fmap-level parallelism when operating at $300 \mathrm{MHz}$. In addition, our architecture can offer an area-speed efficiency 3.77 times higher than XNORBIN, which presents the streaming accelerator.

Table 4. Comparison results of the VLSI implementations.

\begin{tabular}{ccccc}
\hline & {$[30]$} & \multicolumn{2}{c}{ [37] } & Proposed $\left(P_{h w}=\mathbf{1 2 8}\right)$ \\
\hline Technology & $65 \mathrm{~nm}$ & $65 \mathrm{~nm}$ & $22 \mathrm{~nm}$ & $40 \mathrm{~nm}$ \\
\hline Area $\left(10^{3} \mu \mathrm{m}^{2}\right)$ & 540 & 41.2 & 7.6 & 16.49 \\
\hline Normalized Area & 204.49 & 15.60 & 25.12 & 16.49 \\
\hline $\begin{array}{c}\text { Operating } \\
\text { Frequency }\end{array}$ & $156 \mathrm{MHz}$ & \multicolumn{2}{c}{$300 \mathrm{MHz}$} & $300 \mathrm{MHz}$ \\
\hline Peak Performance & $244 \mathrm{Gops} / \mathrm{s}$ & \multicolumn{2}{c}{$66 \mathrm{Gops} / \mathrm{s}$} & $74 \mathrm{Gops} / \mathrm{s}$ \\
\hline $\begin{array}{c}\text { Area Efficiency } \\
\left(\text { Gops/s/ } / A_{\text {norm }}\right)\end{array}$ & 1.19 & 4.23 & 2.63 & 4.49 \\
\hline
\end{tabular}

\section{Conclusions}

In this paper, we proposed a reconfigurable BNN accelerator with an adaptive parallelism scheme. Existing BNN layer accelerators suffer from throughput performance degradation at certain layers in BNN topologies because of their constant data scheduling schemes. On the other hand, streaming architectures, which provide parallelism schemes optimized for each layer, require a large amount of resources and are thus not suitable for edge devices. To overcome such problems, the proposed accelerator analyzes target layer parameters and performs operations with optimal parallelism using reasonable resources. The proposed parallelism schemes consist of fmap-level parallelism combined with an advanced method, which can efficiently cope with certain layers that fmap-level parallelism cannot handle with $100 \%$ utilization. In our performance evaluation for state-ofthe-art BNN topologies, accelerators with the proposed parallelism schemes showed good throughput performance in terms of ops/cycle and low latency compared with existing BNN accelerators. The proposed processor was implemented with 1050 CLBs, 4816 CLB LUTs, and two DSPs on a Xilinx XCZU7EV FPGA device. It offered an area-speed efficiency 9.69 times higher than previous FPGA implementations for BNNs. In addition, it had a logic gate count of $23.37 \mathrm{~K}$ with a die size of $0.016 \mathrm{~mm}^{2}$, and it offered an area-speed efficiency $24 \%$ higher than existing VLSI implementations when operating at $300 \mathrm{MHz}$. 
Author Contributions: J.C. designed the accelerator, performed the simulation and experiment, and wrote the paper. Y.J. (Yongchul Jung) and S.L. implemented the processor and revision of this manuscript. Y.J. (Yunho Jung) conceived of and led the research, analyzed the experimental results, and wrote the paper. All authors read and agreed to the published version of the manuscript.

Funding: This research received no external funding.

Acknowledgments: This work was supported by the Institute of Information \& communications Technology Planning \& Evaluation (IITP) grant funded by the Korean government (MSIT) (No. 2019-0-00056, 2020-0-00201), and the CAD tools were supported by IDEC.

Conflicts of Interest: The authors declare no conflict of interest.

\section{References}

1. Jiao, L.; Zhao, J. A Survey on the New Generation of Deep Learning in Image Processing. IEEE Access 2019, 7, $172231-172263$. [CrossRef]

2. Alom, M.; Tha, T.; Yakopcic, C.; Westberg, S.; Sidike, P.; Nasrin, M.; Hasan, M.; Essen, B.; Awwal, A.; Asari, V. A State-of-the-Art Survey on Deep Learning Theory and Architectures. Electronics 2019, 8, 292. [CrossRef]

3. Hu, R.; Peng, Z.; Ma, J.; Li, W. CNN-Based Vehicle Target Recognition with Residual Compensation for Circular SAR Imaging. Electronics 2020, 9, 555. [CrossRef]

4. He, K.; Zhang, X.; Ren, S.; Sun, J. Deep Residual Learning for Image Recognition. In Proceedings of the IEEE Conference on Computer Vision and Pattern Recognition (CVPR), Las Vegas, NV, USA, 27-30 June 2016; pp. 770-778.

5. Simonyan, K.; Zisserman, A. Very Deep Convolutional Networks for Large-Scale Image Recognition. arXiv 2014, arXiv:1409.1556.

6. Sze, V.; Chen, Y.; Yang, T.; Ember, J. Efficient Processing of Deep Neural Networks: A Tutorial and Survey. Proc. IEEE 2017, 105, 2295-2329. [CrossRef]

7. Zhou, S.; Wu, Y.; Ni, Z.; Zhou, X.; Wen, H.; Zou, Y. DoReFa-Net: Training Low Bitwidth Convolutional Neural Networks with Low Bitwidth Gradients. arXiv 2016, arXiv:1606.06160.

8. Cho, J.; Jung, Y.C.; Lee, S.; Jung, Y.H. VLSI Implementation of Restricted Coulomb Energy Neural Network with Improved Learning Scheme. Electronics 2019, 8, 563. [CrossRef]

9. Zhou, A.; Yao, A.; Guo, Y.; Xu, L.; Chen, Y. Incremental Network Quantization: Towards Lossless CNNs with Low-Precision Weights. arXiv 2017, arXiv:1702.03044.

10. Lee, E.H.; Miyashita, D.; Chai, E.; Murmann, B.; Wong, S. LogNet: Energy-efficient neural networks using logarithmic computation. In Proceedings of the IEEE ICASSP, New Orleans, LA, USA, 5-9 March 2017; pp. 5900-5904.

11. Li, F.; Zhang, B.; Liu, B. Ternary Weight Networks. In Proceedings of the NIPS Workshop Efficient Methods Deep Neural Networks, Barcelona, Spain, 8 December 2016.

12. Jiao, L.; Luo, C.; Cao, W.; Zhou, X.; Wang, L. Accelerating Low bit-width Convolutional Neural Networks with Embedded FPGA. In Proceedings of the 2017 27th International Conference on Field Programmable Logic and Applications (FPL), Ghent, Belgium, 4-8 September 2017.

13. Vita, A.D.; Pau, D.; Benedetto, L.D.; Rubino, A.; Petro, F.; Licciardo, G.D. Low Power Tiny Binary Neural Network with improved accuracy in Human Recognition Systems. In Proceedings of the 2020 23rd Euromicro Conference on Digital System Design (DSD), Kranj, Slovenia, 26-28 August 2020.

14. Vita, A.D.; Russo, A.; Pau, D.; Benedetto, L.D.; Rubino, A.; Licciardo, G.D. A Partially Binarized Hybrid Neural Network System for Low-Power and Resource Constrained Human Activity Recognition. IEEE Trans. CAS 1 2020, 67, 3893-3904.

15. Courbariaux, M.; Bengio, Y.; David, J. BinaryConnect: Training Deep Neural Networks with Binary Weights during propagations. In Proceedings of the NIPS, Montreal, QC, Canada, 7-12 December 2015.

16. Courbariaux, M.; Hubara, I.; Soudry, D.; EI-Yaniv, R.; Bengio, Y. Binarized Neural Networks: Training Deep Neural Networks with Weights and Activations Constrained to +1 or -1 . arXiv 2016, arXiv:1602.02830.

17. Rastegary, M.; Ordonez, V.; Redon, J.; Farhadi, A. XNOR-Net: ImageNet Classification Using Binary Convolutional Neural Networks. arXiv 2016, arXiv:1603.05279.

18. Lin, X.; Zhao, C.; Pan, W. Towards Accurate Binary Convolutional Neural Networks. In Proceedings of the Advances in Neural Information Processing Systems, Long Beach, CA, USA, 4-9 December 2017.

19. Darabi, S.; Belbahri, M.; Courbariaux, M.; Nia, V.P. BNN+: Improved Binary Network Training. In Proceedings of the Learning Representations, Vancouver, BC, Canada, 30 April-3 May 2018; pp. 1-10.

20. Simons, T.; Lee, D. A Review of Binarized Networks. Electronics 2019, 8, 661. [CrossRef]

21. Bethge, J.; Bartz, C.; Yang, H.; Chen, Y.; Meinel, C. MeliusNet: Can Binary Neural Networks Achieve MobileNet-level Accuracy? arXiv 2020, arXiv:2001.05936.

22. Umuroglu, Y.; Fraser, N.J.; Gambardella, G.; Blott, M.; Leong, P.; Jahre, M.; Vissers, K. FINN. In Proceedings of the ACM/SIGDA International Symposium on FPGA, Monterey, CA, USA, 22-24 February 2017; ACM Press: New York, NY, USA, 2017 ; pp. 65-74. 
23. BloTT, M.; Preuber, T.B.; Frased, N.J.; Gambardella, G.; O’brien, K.; Umuroglu, Y.; Leeser, M.; Vissers, K. FINN-R: An End-to-End Deep-Learning Framework for Fast Exploration of Quantized Neural Networks. ACM Trans. Reconfigurable Technol. Syst. 2018, 11, 1-23. [CrossRef]

24. Fiscaletti, G.; Speziali, M.; Stornaiuolo, L.; Santambrogio, M.D.; Sciuto, D. BNNsplit: Binarized Neural Networks for Embedded Distributed FPGA-based Computing Systems. In Proceedings of the 2020 DATE, Grenoble, France, 9-13 March 2020; pp. 975-978.

25. Nurvitadhi, E.; Sheffield, D.; Sim, J.; Mishra, A.; Venkatesh, G.; Marr, D. Accelerating Binarized Neural Networks: Comparison of FPGA, CPU, GPU, and ASIC. In Proceedings of the 2016 International Conference on Field-Programmable Technology (FPT), Xi'an, China, 7-9 December 2016; pp. 77-84.

26. Liang, S.; Yin, S.; Liu, L.; Luk, W.; Wei, S. FP-BNN: Binarized Neural Network on FPGA. Neurocomputing 2012, $275,1072-1086$. [CrossRef]

27. Fu, C.; Zhu, S.; Chen, H.; Koushanfar, F.; Su, H.; Zhao, J. SimBNN: A Similarity-Aware Binarized Neural Network Acceleration Framework. In Proceedings of the IEEE FCCM, San Diego, CA, USA, 28 April-1 May 2019; p. 319.

28. Ghasemzadeh, M.; Samragh, M.; Koushanfar, F. ReBNet: Residual Binarized Neural Network. In Proceedings of the IEEE FCCM, Boulder, CO, USA, 29 April-1 May 2018; pp. 57-64.

29. Geng, T.; Wang, T.; Wu, C.; Yang, C.; Song, S.; Li, A.; Herbordt, M. LP-BNN: Ultra-low-latency BNN Inference with Layer Parallelism. In Proceedings of the 2019 IEEE 30th International Conference on Application-specific Systems, Architectures and Processors (ASAP), New York, NY, USA, 15-17 July 2019; pp. 9-16.

30. Bahou, A.A.; Karunaratne, G.; Andri, R.; Cavigelli, L.; Benini, L. XNORBIN: A 95 TOp/s/W Hardware Accelerator for Binary Convolutional Neural Networks. In Proceedings of the IEEE Symp. COOL CHIPS, Yokohama, Japan, 18-20 April 2018; pp. 1-3.

31. Lin, J.; Xing, T.; Zhao, R.; Zhang, Z.; Srivastava, M.; Tu, Z.; Gupta, R.K. Binarized Convolutional Neural Networks with Separable Filters for Efficient Hardware Acceleration. In Proceedings of the IEEE CVPR, Honolulu, HI, USA, 21-26 July 2017; pp. 27-35.

32. Wang, E.; Davis, J.J.; Cheung, P.Y.K.; Constantinides, G.A. LUTNet: Learning FPGA Configurations for Highly Efficient Neural Network Inference. IEEE Trans. Comput. 2020, 69, 1795-1808. [CrossRef]

33. Lammie, C.; Xiang, W.; Azghadi, M.R. Training Progressively Binarizing Deep Networks using FPGAs. In Proceedings of the IEEE ISCAS, Sevilla, Spain, 12-14 October 2020; pp. 1-5.

34. Yonekawa, H.; Nakahara, H. On-Chip Memory Based Binarized Convolutional Deep Neural Network Applying Batch Normalization Free Technique on an FPGA. In Proceedings of the IEEE IPDPSW, Orlando, FL, USA, 29 May-2 June 2017 ; pp. 98-105.

35. Gu, P.; Ma, H.; Chen, R.; Li, P.; Xie, S.; Wang, D. FBNA: A Fully Binarized Neural Network Accelerator. In Proceedings of the 2018 28th International Conference on Field Programmable Logic and Applications (FPL), Dublin, Ireland, 27-31 August 2018; pp. 51-54.

36. Zhou, Y.; Redkar, S.; Huang, X. Deep Learning Binary Neural Network on an FPGA. In Proceedings of the IEEE MWSCAS, Boston, MA, USA, 6-9 August 2017; pp. 281-284.

37. Conti, F.; Schiavone, D.; Benini, L. XNOR Neural Engine: A Hardware Accelerator IP for 21.6fj/op Binary Neural Network Inference. IEEE Trans. CAD 2018, 37, 2940-2951. [CrossRef]

38. Rusci, M.; Cavigelli, L.; Benini, L. Design Automation for Binarized Neural Networks: A Quantum Leap Opportunity? In Proceedings of the IEEE ISCAS, Florence, Italy, 27-30 May 2018.

39. Jung, Y.C.; Cho, J.; Lee, S.; Jung, Y.H. Area-Efficient Pipelined FFT Processor for Zero-Padded Signals. Electronics 2019,8 , 1397. [CrossRef] 